\title{
Corrigendum: Structure of a prokaryotic fumarate transporter reveals the architecture of the SLC26 family
}

Eric R Geertsma, Yung-Ning Chang, Farooque R Shaik, Yvonne Neldner, Els Pardon, Jan Steyaert \& Raimund Dutzler Nat. Struct. Mol. Biol. 22, 803-808 (2015); published online 14 September 2015; corrected after print 12 April 2016

In the version of this article initially published, the Protein Data Bank accession code for the coordinates and structure factors for SLC26Dg ${ }^{\Delta T A S}$ (PDB 5IOF) was not included. The error has been corrected in the HTML and PDF versions of the article. 